\title{
Predictors of treatment response following aspiration sclerotherapy of hepatic cysts: an international pooled analysis of individual patient data
}

\author{
Titus F. M. Wijnands ${ }^{1}$ - Maxime Ronot ${ }^{2}$ - Tom J. G. Gevers ${ }^{1} \cdot$ Julie Benzimra $^{2}$ • \\ Leo J. Schultze Kool ${ }^{3}$. Valérie Vilgrain ${ }^{2}$ Joost P. H. Drenth ${ }^{1}$
}

Received: 17 November 2015 /Revised: 29 February 2016/Accepted: 11 April 2016/Published online: 14 May 2016

(C) The Author(s) 2016. This article is published with open access at Springerlink.com

\begin{abstract}
Objectives To identify predictive variables of treatment response following aspiration sclerotherapy of large symptomatic hepatic cysts.

Methods We collected individual patient data from two tertiary referral centres and included all patients treated with aspiration sclerotherapy of a large $(>5 \mathrm{~cm})$, symptomatic hepatic cyst. At six months, clinical response was defined as complete or incomplete. Secondary, suboptimal technical response was defined as lower quartile of cyst reduction. Predictive variables of clinical and technical response were analyzed by logistic regression analysis.

Results We included 86 patients ( $58 \pm 10$ years; female $90 \%$ ). Complete clinical response rate was $55 \%$. Median cyst diameter and volume reduction were $71 \%$ (IQR 50-87\%) and $98 \%$ (IRQ 88-100\%), respectively. Patients with complete clinical response had a significantly higher cyst reduction compared to incomplete responders (OR 1.02, $95 \% \mathrm{CI}$ 1.00-1.04). Aspiration of haemorrhagic cyst fluid (OR 4.39,
\end{abstract}

Electronic supplementary material The online version of this article (doi:10.1007/s00330-016-4363-x) contains supplementary material, which is available to authorized users.

Joost P. H. Drenth

joostphdrenth@cs.com

1 Department of Gastroenterology and Hepatology, Radboud University Medical Center, P.O. Box 9101, code 455, 6500 HB Nijmegen, The Netherlands

2 Department of Radiology, Beaujon University Hospitals Paris Nord Val de Seine, Clichy, France

3 Department of Radiology, Radboud University Medical Center, Nijmegen, The Netherlands
$95 \%$ CI 1.34-14.39) or a lower cyst reduction at one month (OR 1.06, $95 \%$ CI 1.02-1.10) was associated with a suboptimal technical response at six months.

Conclusion Complete clinical response is associated with effective cyst reduction. Aspiration of haemorrhagic cyst fluid or a restricted diameter reduction at one month predicts a suboptimal technical treatment response, however, these variables did not predict symptom disappearance.

Key Points

- Aspiration sclerotherapy of hepatic cysts shows excellent clinical and technical efficacy.

- Optimal clinical responders have a markedly higher cyst reduction.

- Haemorrhagic aspirate and a strong fluid reaccumulation predict suboptimal cyst reduction.

Keywords Hepatic cyst · Polycystic liver disease · Interventional $\cdot$ Drainage $\cdot$ Sclerotherapy

$\begin{array}{ll}\text { Abbreviations } \\ \text { CT } & \text { Computed tomography } \\ \text { MRI } & \text { Magnetic resonance imaging } \\ \text { PLD } & \text { Polycystic liver disease } \\ \text { US } & \text { Ultrasound }\end{array}$

Introduction

Hepatic cysts are fluid-filled cavities that arise from congenital malformations of biliary ducts [1]. The estimated prevalence of these benign, non-parasitic lesions is $2-18 \%$ in the general population [2-4]. Hepatic cysts occur solitary or as multiple lesions in the context of polycystic liver disease (PLD) [5]. Aspiration sclerotherapy is a minimally invasive treatment 
option and serves as first-choice treatment for symptomatic hepatic cysts [6-8]. Aspiration sclerotherapy combines percutaneous drainage of cyst fluid with subsequent instillation of a sclerosing agent that destroys the inner cyst lining. This treatment is effective and safe [9-14]. There are two components that are important to the perceived success of aspiration sclerotherapy treatment. First, clinical response as experienced by the patient, and second, technical response in terms of reduction of the treated cyst. It is not well understood which factors determine clinical success or are important for technical response. Due to the infrequency of large symptomatic hepatic cysts, published studies are relatively small, which is an obstacle on the road to identification of predictive factors. In this study, we collected individual patient data from two observational cohort studies with comparable treatment protocols and follow-up measurements. The goal of this study was to identify variables that predict response of aspiration sclerotherapy of large symptomatic hepatic cysts.

\section{Materials and methods}

\section{Patients and study design}

We performed a pooled analysis of data collected from patients that consecutively underwent aspiration sclerotherapy in two tertiary referral centres: Radboud University Medical Center, Nijmegen, the Netherlands (centre 1) and University Hospitals Paris Nord Val de Seine, Beaujon, Clichy, France (centre 2) (Table 1). Centre 1 prospectively included patients treated within a timeframe of June 2012 to March 2014, centre 2 retrospectively included patients treated from December 2003 to September 2011. Within this timeframe the treatment protocol remained unchanged. For this analysis we included a cohort (centre 2) that was previously published to report efficacy of aspiration sclerotherapy [13]. In the current study, we extended our data by pooling this sample with a second prospective cohort (centre 1). By this, we created a large sample size that was needed to evaluate predictors of treatment response.
Included patients were aged over 18 years and underwent aspiration sclerotherapy of a simple, symptomatic, solitary or dominant hepatic cyst larger than $5 \mathrm{~cm}$ (Fig. 1). A simple cyst was diagnosed by ultrasound (US), computed tomography (CT), or magnetic resonance imaging (MRI) based on the following criteria: a well-circumscribed anechoic lesion with increased posterior echo enhancement and no evidence of mural nodularity on US; a water-density ( -10 to $10 \mathrm{HU}$ ) lesion with sharply defined margins and smooth thin walls with no contrast enhancement or septations on CT; or a homogeneous lesion, hypointense on T1-weighted images and hyperintense on T2-weighted images without contrast enhancement or septations on MRI $[15,16]$. A history of a previous cyst haemorrhage was not considered an exclusion criterion. The diagnosis of a haemorrhagic cyst was based on pre-defined criteria [16]. If deemed necessary, serology and/or more extensive imaging was applied to rule out hydatid or (pre-)malignant lesions. Parasitic or neoplastic cysts were not treated by aspiration sclerotherapy. In PLD patients ( $>20$ cysts), the largest cyst was regarded as dominant cyst. A patient was excluded from analysis if the treated cyst could not be identified following treatment or was lost to follow-up. If a patient underwent two or more aspiration sclerotherapy procedures of the same cyst, only the first procedure was included in this analysis. The institutional review board of both participating centres approved this study and requirement for informed consent was waived.

\section{Aspiration sclerotherapy}

Patients were informed about possible related risks and agreed to undergo aspiration sclerotherapy. In both centres, patients underwent aspiration sclerotherapy of a single hepatic cyst following a standardized single-session percutaneous ethanol sclerotherapy protocol [13]. The intervention was performed with local anaesthesia and conscious sedation in centre 1, and under general anaesthesia in centre 2 . The cyst was localized by US and punctured with a 5-French pigtail catheter (centre 1: Cook Medical, Bloomington, United States of America; centre 2: Cordis Corporation, Bridgewater, United States of
Table 1 Design characteristics of included studies

\begin{tabular}{lll}
\hline & Centre 1 & Centre 2 \\
\hline Number of patients & 29 & 57 \\
Design & Prospective cohort & Retrospective cohort \\
Type treatment & Single session, ethanol & Single session, ethanol \\
Population & Solitary cysts and PLD & Solitary cysts and PLD \\
Inclusion criteria & $>18$ y; simple, non-parasitic cyst & $>18$ y; simple, non-parasitic cyst \\
Endpoints & Clinical and technical outcome, safety & Clinical and technical outcome, safety \\
Timepoints & Baseline, one, and six months & Baseline, one, six, and 12 months \\
\hline
\end{tabular}

Abbreviations: PLD, polycystic liver disease; y: years 

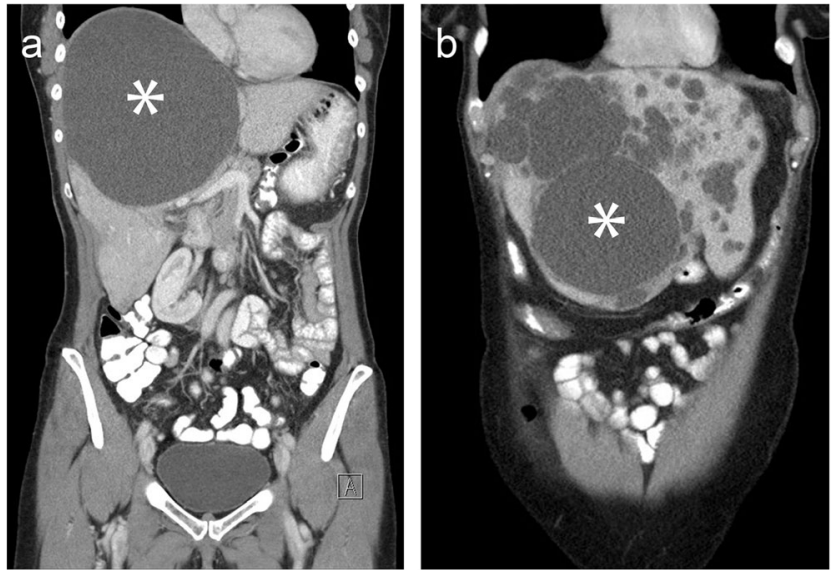

Fig. 1 Computed tomography demonstrating (asterisk) a solitary (a) and dominant hepatic cyst in polycystic liver disease (b)

America) to perform complete fluid drainage of the hepatic cyst (Fig. 2). Aspirated fluid was classified as clear or haemorrhagic (red or brown) fluid and evaluated by microscopy. Subsequently, we performed fluoroscopy following instillation of contrast fluid (centre 1: Iomeron 300, Bracco Imaging, Konstanz, Germany; centre 2: Iobitridol, xenetix 350 , Guerbet, France) to rule out leakage or communication with vasculature or the biliary tree (Fig. 2). Sclerotherapy was performed by injecting 96-100\% ethanol (centre 1: up to $50 \mathrm{~mL}, 10$ minutes; centre 2: up to $150 \mathrm{~mL}, 60$ minutes) in the cyst. Following sclerotherapy, all ethanol was re-aspirated and the catheter was removed from the patient. Patients were discharged one day following the procedure.

\section{Baseline and follow-up study visits}

At baseline and at one and six months following aspiration sclerotherapy, the patient visited the outpatient clinic to evaluate clinical and technical parameters. In both centres, overall clinical response was assessed at six months by standardized instruments and defined as "disappearance", "reduction", "no change", or "aggravation" of symptoms
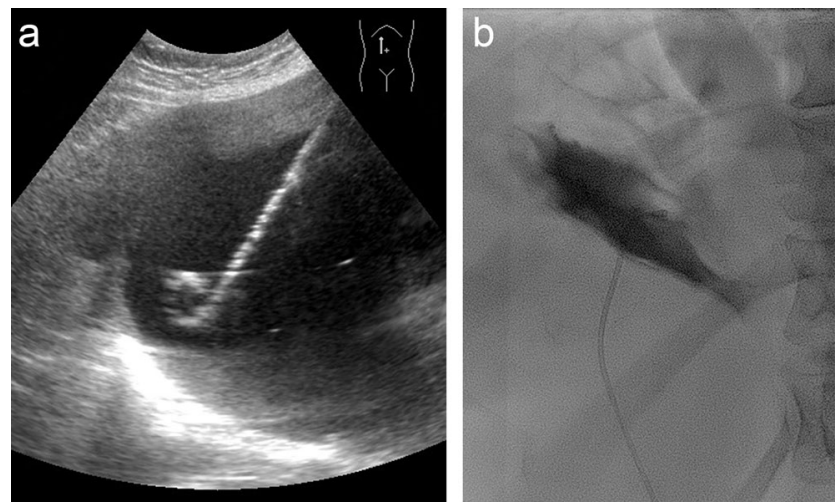

Fig. 2 Ultrasonography-guided placement of pigtail catheter to perform aspiration (a) followed by fluoroscopy demonstrating a collapsed hepatic cyst without leakage (b)
$[13,17]$. If a patient had not completed the questionnaire, we retrospectively reviewed the patients' clinical chart $(n=10)$. Detailed information of symptom assessment can be found in the supplemental material.

The maximal cyst diameter of the treated cyst was measured during all visits (Fig. 3). Subsequently, we estimated cyst volume by multiplying available orthogonal diameters by 0.523 using the ellipsoid volume formula $(\mathrm{d} 1 * \mathrm{~d} 2 * \mathrm{~d} 3 * 0.523)$. If an orthogonal diameter was missing, we substituted this value by the maximal diameter. In centre 1, all measurements were performed by US (Acuson $\mathrm{X} 150^{\mathrm{TM}}$, Siemens Healthcare, Erlangen, Germany) by the same investigator. In centre 2, US (Aixplorer ultrasound system, SuperSonic Imagine, Aix-en-Provence, France; Aplio, Toshiba Medical system, Tokyo, Japan), CT (Advantage LightSpeed VCT, GE Healthcare, Milwaukee, United States of America), or MRI (1.5-T imager, interna, Philips Healthcare, Best, the Netherlands) were applied and measured by two investigators. Both centres monitored adverse events until six months following treatment.

\section{Outcome variables}

The primary outcome was the overall clinical response at six months following treatment. To predict optimal clinical success, we divided clinical outcome into two groups: complete clinical responders that reported "disappearance" or incomplete clinical responders that reported "reduction", "no change" or "aggravation" of symptoms.

As a secondary outcome, technical response was evaluated by the proportional cyst reduction at six months. From a technical point of view, we were mainly interested in patients with a low (suboptimal) outcome to identify factors that are associated with lower efficacy of the procedure. To do so, we grouped technical response into patients with or without a suboptimal response, defined by a cyst diameter reduction in

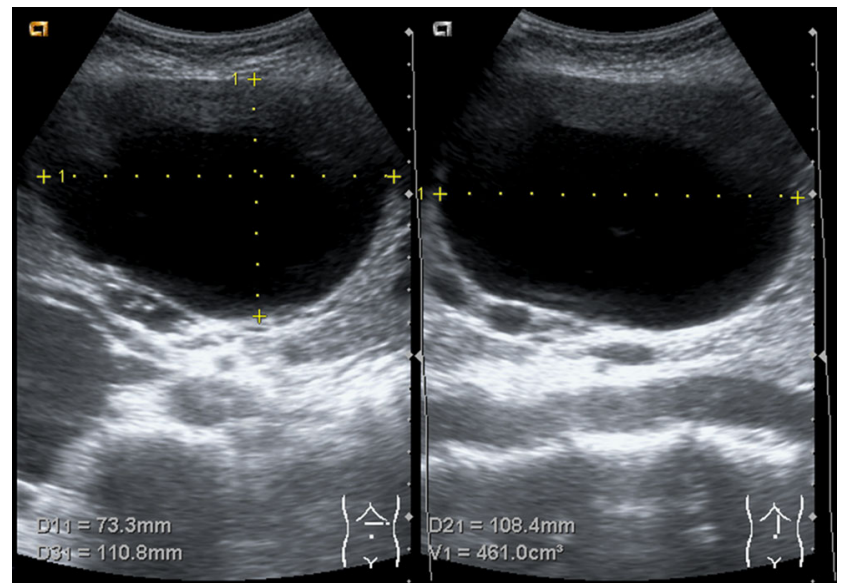

Fig. 3 Hepatic cyst diameter measurement by ultrasonography; transversal (left) and coronal (right) plane to estimate hepatic cyst volume 
the lower quartile of the total sample. Secondly, we repeated this evaluation with cyst volume reduction measurements.

\section{Predicting variables}

The following variables were selected for analysis: age at treatment (years), gender, PLD (defined as $>20$ cysts), previous drainage of the treated cyst, baseline cyst diameter $(\mathrm{cm})$, location of the treated cyst (right or left liver lobe), haemorrhagic aspect of aspirated cyst fluid, volume of injected ethanol (mL), and proportional diameter reduction (\%) at one and six month(s) following aspiration sclerotherapy.

\section{Data collection}

Both centres completed an electronic form containing all variables of interest. A pooled dataset was created by the initiating investigator. If possible, data inconsistencies were corrected in agreement with both centres.

\section{Statistical analysis}

Baseline characteristics are given in mean $( \pm \mathrm{SD})$ or median $( \pm$ IQR) according to distribution. Patients characterized as complete clinical responder were compared with patients with an incomplete clinical response on predefined predicting variables by univariate analysis. Similarly, we compared patients with or without a suboptimal technical response. Variables were selected as fixed effects in logistic regression analysis (generalized linear mixed models) if a $p$-value $<0.2$ was found. To identify predictors of clinical and technical response, we used backward selection by excluding variables with a $p$-value $>0.05$ in the multivariate analysis. The variable centre retained in univariate and multivariate analysis as a random effect to correct for possible differences between centres. Within groups of symptomatic relief, we compared proportional cyst diameter at six months by the non-parametric Kruskal-Wallis test. In addition, we explored whether a suboptimal technical response was associated with clinical response using the Pearson's chi-square test. Finally, to confirm our findings, we repeated all uni- and multivariate analyses using cyst volume measurements instead of diameter measurements. Statistical analyses were performed using IBM SPSS software, version 20.0. $P$-values were two-tailed and a value $<0.05$ was considered statistically significant.

\section{Results}

\section{Baseline characteristics}

A total of 93 patients was retrieved from both centres. Seven patients were excluded from analysis (Fig. 4). Reasons for

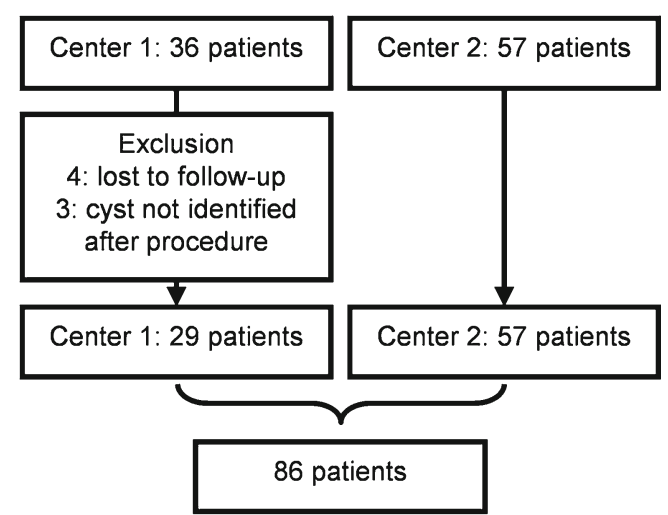

Fig. 4 Overview of included patients

exclusion were loss to follow-up ( $n=4)$ or inability to identify the treated cyst from other surrounding cysts following aspiration sclerotherapy $(n=3)$. This resulted in a pooled sample of 86 patients with a mean age at treatment of 57.8 (range 28 $80)$ years (Table 2). Female patients $(n=77,89.5 \%)$ had a mean age of 57.0 (range $28-80)$ years, male patients $(n=9)$ had a mean age of $65.0(57-79)$ years.

Cysts were most frequently localized in the right hepatic lobe $(69.8 \%)$. PLD was present in $38.4 \%$. The median maximal diameter at baseline of the treated hepatic cyst was $11.0 \mathrm{~cm}(9.3-15.0 \mathrm{~cm})$ corresponding with a median estimated baseline volume of $696 \mathrm{~mL}$ (381-1584 mL). Aspect of cyst aspirate was assessed in 84 cases: $52(61.9 \%)$ aspirates were classified as clear and $32(38.1 \%)$ aspirates contained haemorrhagic constituents. This was confirmed by microscopic evaluation.

\section{Overall clinical and technical response}

At six months, 47 patients (54.7\%) reported disappearance of symptoms and were defined as complete clinical responders.

Table 2 Demographics $(n=86)$

\begin{tabular}{lc}
\hline Age at treatment, mean (SD) & $57.8(10.2)$ \\
\hline Female gender, $n(\%)$ & $77(89.5 \%)$ \\
PLD, $n(\%)$ & $33(38.4 \%)$ \\
Previous drainage treated cyst, $n(\%)$ & $19(22.1 \%)$ \\
Location cyst in right liver lobe, $n(\%)$ & $60(69.8 \%)$ \\
Cyst diameter baseline, median (IQR) & $11.0(9.3-15.0)$ \\
Cyst volume baseline, median (IRQ) & $696(381-1584)$ \\
Volume aspirate, median (IQR) & $500(300-1200)$ \\
Haemorrhagic aspect of cyst fluid, $n(\%)$ & $32(38.1 \%)^{1}$ \\
Volume of ethanol, median (IRQ) & $70(50-110)$ \\
\hline
\end{tabular}

${ }^{1}$ Cyst fluid aspect was not characterized in two patients. Age is presented in years, diameters in centimeters, and volumes in milliliters. Abbreviations: PLD, polycystic liver disease; SD, standard deviation; IQR, interquartile range 
Thirty patients $(34.9 \%)$ reported reduction, and nine patients $(10.4 \%)$ had no change of symptoms following aspiration sclerotherapy and were collectively defined as incomplete clinical responders. In total, $89.6 \%$ of patients had either disappearance or reduction of symptoms. No patients reported aggravation of symptoms.

Median cyst diameter decreased from $11.0 \mathrm{~cm}$ (9.3$15.0 \mathrm{~cm})$ to $8.6 \mathrm{~cm}(6.0-11.0 \mathrm{~cm})$ one month following aspiration sclerotherapy, corresponding to a median proportional reduction of $29.7 \%$ (17.4-39.2 \%). Cyst diameter regression continued to a median cyst diameter of $3.5 \mathrm{~cm}(1.0-6.4 \mathrm{~cm})$ six months following aspiration sclerotherapy, corresponding to a proportional reduction of $70.7 \%$ (50.0-87.2\%). In line, median proportional cyst volume regressed by $68.0 \%(40.4-78.4 \%)$ to $264 \mathrm{~mL}$ (103-605 mL) in the first month. At six months, median volume regressed to $22 \mathrm{~mL}(1-101 \mathrm{~mL})$ corresponding with a proportional reduction of $97.8 \%$ (87.5-99.8\%). Overall, centre 2 reported a higher cyst reduction at six months than centre 1: $99 \%$ (94-100) vs $92 \%$ (68-98\%), respectively.

\section{Predictors of complete clinical response}

After univariate analysis, we selected location of the treated cyst, volume of injected ethanol, proportional diameter reduction at one month, and proportional diameter reduction at six months for subsequent regression analysis (Table 3 ). Multivariate analysis identified that an increased proportional diameter reduction at six months was associated with a complete clinical response (OR 1.02, $95 \%$ CI 1.00-1.04, $p=0.046$ ). When we replaced proportional diameter reduction at six months for volume measurements, statistical significance was lost (OR 1.02, $95 \%$ CI 0.99-1.05, $p=0.665$,
Supplementary Table 1). We observed no significant centre effect $(p=0.13)$.

Further explorative analysis revealed that proportional diameter reduction at six months was $78.6 \%(60.0-92.9 \%)$ in patients with symptom disappearance, $62.9 \%(43.4-82.8 \%)$ in patients with symptom reduction, and $50 \%(24.5-66.4 \%)$ in patients who reported no change of symptoms ( $p$-value $=0.01$, Fig. 5). In accordance with our diametric analysis, patients with symptom disappearance showed a statistically significant higher median volume reduction at six months of $99.0 \%$ (94.1-100\%), compared to $94.4 \%$ (79.1-99.5\%) and $87.5 \%(60.6-95.9 \%)$ in patients with reduction or no change of symptoms, respectively ( $p$-value $=0.02)$.

\section{Predictors of suboptimal technical response}

The lower quartile in technical response $(n=22)$ was defined by a proportional diameter reduction of $<50.0 \%$ and a volume reduction of $<87.5 \%$, characterized as suboptimal technical response.

Baseline cyst diameter, proportional cyst diameter reduction after one month, and aspect of cyst fluid were selected for subsequent regression analysis (Table 4). By multivariate analysis, we identified that patients with lower diameter reduction at one month had higher risk of having a suboptimal technical response at six months (OR 1.06, $95 \%$ CI 1.02-1.10, $p<0.01)$. In addition, cysts containing haemorrhagic fluid were associated with a suboptimal technical response (OR $4.39,95 \%$ CI 1.34-14.39, $p=0.02$ ). We confirmed our findings when diameter outcome and predictors were replaced by volume measurements (Supplementary Table 2). A suboptimal technical response was predicted by a lower volume reduction at one month (OR 1.04, $95 \%$ CI 1.02-1.06, $p<0.01$ ) and haemorrhagic cyst fluid (OR 4.78, $95 \%$ CI 1.38-16.54,

Table 3 Predictors of complete clinical response, univariate and multivariate analysis

\begin{tabular}{|c|c|c|c|c|c|}
\hline Independent variable & $\begin{array}{l}\text { Complete } \\
\text { response }(n=47)\end{array}$ & $\begin{array}{l}\text { Incomplete } \\
\text { response }(n=39)\end{array}$ & $\begin{array}{l}\text { Univariate } \\
\text { analysis, } p \text {-value }\end{array}$ & $\begin{array}{l}\text { Multivariate analysis, } \\
\text { Odds ratio (95\% CI) }\end{array}$ & $\begin{array}{l}\text { Multivariate } \\
\text { analysis, } p \text {-value }\end{array}$ \\
\hline Age at treatment, mean (SD) & $57.9(10.3)$ & $57.7(10.3)$ & 0.962 & & \\
\hline Female gender, $n(\%)$ & $44(93.6)$ & $33(84.6)$ & 0.227 & & \\
\hline PLD, $n(\%)$ & $16(34.0)$ & $17(43.6)$ & 0.402 & & \\
\hline Previous drainage of treated cyst, $n(\%)$ & $9(23.1)$ & $10(21.3)$ & 0.889 & & \\
\hline Baseline cyst diameter, mean (SD) & $11.8(3.5)$ & $12.7(4.0)$ & 0.260 & & \\
\hline Location cyst in right liver lobe, $n(\%)$ & $30(63.8)$ & $30(76.9)$ & 0.179 & $1.74(0.62-4.86)$ & 0.288 \\
\hline Haemorrhagic aspect of cyst fluid, $n(\%)^{1}$ & $17(63.0)$ & $15(60.5)$ & 0.940 & & \\
\hline Volume of ethanol, median (IQR) & $80.0(40.0-110.0)$ & $50.0(50.0-110.0)$ & 0.143 & $0.99(0.97-1.01)$ & 0.319 \\
\hline $\begin{array}{l}\text { Proportional diameter reduction one } \\
\text { month, median (IQR) }\end{array}$ & $32.1(21.4-45.5)$ & $27.6(11.8-36.7)$ & 0.118 & $1.01(0.98-1.04)$ & 0.425 \\
\hline $\begin{array}{l}\text { Proportional diameter reduction six } \\
\text { months, median (IQR) }\end{array}$ & $78.6(60.0-92.3)$ & $59.1(39.2-81.3)$ & 0.046 & $1.02(1.00-1.04)$ & 0.046 \\
\hline
\end{tabular}

\footnotetext{
${ }^{1}$ Cyst fluid aspect was not characterized in two patients. Age is presented in years, diameter in centimeters, volume in milliliters and proportional reductions in percentages. Abbreviations: PLD, polycystic liver disease; SD, standard deviation; IQR, interquartile range
} 


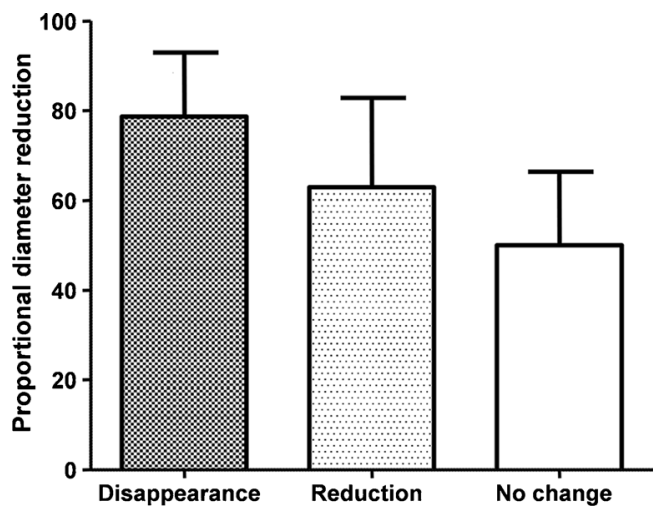

Fig. 5 Proportional cyst diameter reduction within groups of symptom relief at six months

$p=0.01)$. Again, we found no significant centre effect $(p=0.06)$. Finally, explorative analysis revealed that 8 of 22 $(36.4 \%)$ patients with a suboptimal technical response had complete clinical response, compared to 39 of 64 (60.9\%) patients without a suboptimal technical response, a difference that was statistically significant $(p=0.046)$.

\section{Safety}

Overall, nine adverse events (10.7 \%) occurred. Four patients developed an adverse event immediately after treatment, these included: hypotension $(n=2)$, transient fever $(n=1)$, and a cyst haemorrhage $(n=1)$. During follow-up, four patients reported pain between 2-24 days after treatment, in three of these patients a cyst haemorrhage was suspected based on clinical and radiological presentation. Finally, one patient developed an hepatic cyst infection $(n=1)$, which was treated adequately by antibiotic treatment. The remaining adverse events were managed by conservative treatment. All adverse events resolved without sequelae.

\section{Discussion}

The primary finding of this study is that a strong hepatic cyst reduction following aspiration sclerotherapy predicts complete clinical response. We also identified that haemorrhagic cyst aspirate and a limited cyst size reduction at one month predicted suboptimal long-term cyst reduction but did not affect clinical response.

Clinical efficacy in this pooled cohort was high as $89.6 \%$ of treated patients reported reduction of symptoms while symptoms disappeared completely in $54.7 \%$. The literature contains four large studies that assessed symptomatic response following aspiration sclerotherapy with various sclerosing agents. Similar to our results, these studies reported reduction of symptoms in 72.0-92.2 \%, while 46.1-85.0 \% had complete resolution of symptoms $[7,10,18,19]$. However, these studies failed to investigate which factors contribute to clinical treatment response. We used the model of an individual patient data analysis with data coming from two independent cohorts, to demonstrate that a significant reduction of the treated cyst was associated to a better overall clinical response. Indeed, patients with a cyst diameter reduction over $50 \%$ were more likely to have complete clinical response. This underlines that effective technical success is needed to increase the patients' chance to benefit of treatment.

We observed a median proportional diameter reduction of $71 \%$ at six months following aspiration sclerotherapy, corresponding with a volume reduction of $98 \%$. This strong reduction rate is comparable to other studies that evaluated long-term technical success of aspiration sclerotherapy. These studies achieved a proportional diameter reduction ranging from 54.8-85.7\% after 12-48 months [7, 20-22]. Correspondingly, studies that reported efficacy in terms of proportional volume reduction ranged from 90.8-98.9\% following 12-30 months [18, 23-25].

Table 4 Predictors of suboptimal technical response, univariate and multivariate analysis

\begin{tabular}{|c|c|c|c|c|c|}
\hline Independent variable & $\begin{array}{l}\text { Suboptimal } \\
\text { response }(n=22)\end{array}$ & $\begin{array}{l}\text { No suboptimal } \\
\text { response }(n=64)\end{array}$ & $\begin{array}{l}\text { Univariate } \\
\text { analysis, } p \text {-value }\end{array}$ & $\begin{array}{l}\text { Multivariate analysis, } \\
\text { odds ratio }(95 \% \mathrm{CI})\end{array}$ & $\begin{array}{l}\text { Multivariate } \\
\text { analysis, } p \text {-value }\end{array}$ \\
\hline Age at treatment, mean (SD) & $60.6(9.8)$ & $56.8(10.2)$ & 0.095 & $1.04(0.98-1.10)$ & 0.202 \\
\hline Female gender, $n(\%)$ & $19(86.4)$ & $58(90.6)$ & 0.712 & & \\
\hline $\mathrm{PLD}, n(\%)$ & $9(40.9)$ & $24(34.5)$ & 0.860 & & \\
\hline Previous drainage of treated cyst, $n(\%)$ & $4(18.2)$ & $15(23.4)$ & 0.536 & & \\
\hline Baseline cyst diameter, mean (SD) & $13.6(4.4)$ & $11.7(3.4)$ & 0.046 & $1.10(0.94-1.29)$ & 0.239 \\
\hline Location cyst in right liver lobe, $n(\%)$ & $16(72.7)$ & $44(68.8)$ & 0.692 & & \\
\hline Haemorrhagic aspect of cyst fluid, $n(\%)^{1}$ & $13(59.1)$ & $19(29.7)$ & 0.034 & $4.39(1.34-14.39)$ & 0.015 \\
\hline Volume of ethanol, median (IQR) & $50.0(47.5-110.0)$ & $50.0(50.0-110.0)$ & 0.320 & & \\
\hline $\begin{array}{l}\text { Proportional diameter reduction one } \\
\text { month, median (IQR) }\end{array}$ & $17.1(4.1-36.7)$ & $32.0(24.6-45.2)$ & 0.009 & $1.06(1.02-1.10)$ & 0.007 \\
\hline
\end{tabular}

\footnotetext{
${ }^{1}$ Cyst fluid aspect was not characterized in two patients. Age is presented in years, diameter in centimeters, volume in milliliters and proportional reductions in percentages. Abbreviations: $\mathrm{PLD}$, polycystic liver disease; SD, standard deviation; IQR, interquartile range
} 
Sclerotherapy of hepatic cysts containing haemorrhagic fluid is not contraindicated and leads to reduction of the cyst [13]. Nonetheless, we found that drainage of haemorrhagic cyst fluid predicted an increased risk of a suboptimal technical treatment response. This may be the result of several factors. Due to increased viscosity, residues of haemorrhagic cyst fluid may remain in the cyst following drainage, leading to a diluted concentration of the sclerosing agent $[7,26]$. Alternatively, clots or debris in the cyst may prevent contact with the cyst wall [10]. A spontaneous cyst bleeding is estimated to occur in around $8 \%$ of hepatic cysts [27]. Remarkably, 32 (38\%) patients in our sample had cyst fluid containing haemorrhagic constituents. This relatively large proportion of haemorrhagic cyst fluid in treated patients may result of the symptomatic course following a cyst bleed, ultimately leading to treatment of the cyst. In this study, haemorrhagic content was not associated to a reduced clinical response. The present results validate the use of aspiration sclerotherapy in this subgroup of patients.

Furthermore, we found that a lower cyst reduction at one month predicted a suboptimal technical response at six months. Intracystic fluid re-accumulation directly following aspiration sclerotherapy is a well-known phenomenon that leads to a restricted cyst reduction in the first week(s) [28]. Although temporary, the cyst may relapse completely following aspiration sclerotherapy [24]. Previous studies speculated that secretory function of cyst-lining epithelial cells may persist following sclerotherapy; others considered fluid re-accumulation as a component of inflammatory response [7, 29]. Although transient in most cases, we found that a strong relapse predicted a suboptimal long-term cyst reduction.

Apart from aspiration sclerotherapy, surgical fenestration is often advocated as a primary treatment option for hepatic cysts. In a review of 43 studies (311 patients) evaluating efficacy and safety of cyst fenestration, reduction of symptoms was found in $92 \%$ of treated patients [8]. This clinical response is consistent with our results. However, $22 \%$ of laparoscopic procedures were converted to open fenestration and overall morbidity following cyst fenestration was reported in $23 \%$ of cases, whereas in our study $11 \%$ of patients experienced an adverse event. Our results suggest that aspiration sclerotherapy has comparable clinical efficacy with lower morbidity rates compared to its surgical alternative.

In this study, we confirm that aspiration sclerotherapy is highly effective in reducing symptoms. Therefore, we advocate that aspiration sclerotherapy should be primary treatment for patients with a symptomatic, solitary, or dominant hepatic cyst. In patients with haemorrhagic cyst fluid aspirate or a strong fluid reaccumulation following aspiration sclerotherapy, we advise increased attention. If symptoms recur, initiation of a subsequent aspiration sclerotherapy or referral for surgical treatment may be favourable.

The main strength of this study is that we pooled results of two similar studies from different countries and thereby increased the generalizability of our results. Moreover, this relatively large sample size enabled us to evaluate predictors of treatment response. Some limitations come with this study. Firstly, symptomatic change was evaluated by two different questionnaires. A detailed evaluation of specific symptoms could not be performed due to differences between these instruments. However, as both instruments assessed overall symptomatic response in a comparable standardized manner, we were able to pool results of overall clinical response and believe that possible introduced bias is limited. Secondly, due to small numbers of patients without reduction of symptoms, we grouped patients with "reduction" of symptoms and "no change" as incomplete clinical responder. As a result, we were unable to evaluate predicting variables of clinical nonresponders. Furthermore, our follow-up extends over a period of six months and should ideally be validated over a longer period. However, we regard six months as sufficient as the most prominent cyst regression occurs within these first months and sustains thereafter [24]. Moreover, cyst diameter measurements were performed by different imaging modalities and investigators between centres; this could have led to differences in outcome measurements. Finally, duration of ethanol instillation differed between centre 1 and centre 2 . As this was completely co-linear to centre, we could not include this variable in our analysis. A prolonged exposure of the sclerosing agent to the cyst wall may improve efficacy of treatment. On the other hand, previous studies stated that ethanol eliminates epithelial cells within minutes $[24,30]$. The impact of duration of sclerotherapy on treatment efficacy remains to be tested.

To conclude, aspiration sclerotherapy of hepatic cysts effectively reduces symptoms in the vast majority of patients. Chance of complete clinical response is increased in patients with higher proportional cyst reduction. We identified that a limited cyst reduction in the first month and the presence of haemorrhagic cyst fluid predict a suboptimal technical treatment response, however, these variables did not predict symptom disappearance.

Acknowledgments The authors thank Wietske Kievit from the Department of Health Evidence Radboud university medical center, Nijmegen, the Netherlands, for her expert methodological advice. We also thank Victor Sidy, architectural researcher and designer, Phoenix, Arizona, United States, for English language editing. The scientific guarantor of this publication is Joost Drenth.

The authors of this manuscript declare no relationships with any companies, whose products or services may be related to the subject matter of the article. The authors state that this work has not received any funding. Wietske Kievit from the Department of Health Evidence Radboud University Medical Center, Nijmegen, the Netherlands kindly provided statistical advice for this manuscript.

Institutional Review Board approval was obtained. Written informed consent was waived by the Institutional Review Board. Some study subjects have been previously reported in Benzimra et al. Eur Radiol. 10. 1007/s00330-014-3117-x. Methodology: Pooled analysis of prospective and retrospective cohort, observational, multicenter study. 
Open Access This article is distributed under the terms of the Creative Commons Attribution 4.0 International License (http:// creativecommons.org/licenses/by/4.0/), which permits unrestricted use, distribution, and reproduction in any medium, provided you give appropriate credit to the original author(s) and the source, provide a link to the Creative Commons license, and indicate if changes were made.

\section{References}

1. Everson GT, Taylor MR, Doctor RB (2004) Polycystic disease of the liver. Hepatology 40:774-782

2. Gaines PA, Sampson MA (1989) The prevalence and characterization of simple hepatic cysts by ultrasound examination. Br J Radiol 62:335-337

3. Larssen TB, Rorvik J, Hoff SR, Horn A, Rosendahl K (2005) The occurrence of asymptomatic and symptomatic simple hepatic cysts. A prospective, hospital-based study. Clin Radiol 60:1026-1029

4. Carrim ZI, Murchison JT (2003) The prevalence of simple renal and hepatic cysts detected by spiral computed tomography. Clin Radiol 58:626-629

5. Gevers TJ, Drenth JP (2013) Diagnosis and management of polycystic liver disease. Nat Rev Gastroenterol Hepatol 10:101-108

6. Erdogan D, van Delden OM, Rauws EA et al (2007) Results of percutaneous sclerotherapy and surgical treatment in patients with symptomatic simple liver cysts and polycystic liver disease. World J Gastroenterol 13:3095-3100

7. Tikkakoski T, Makela JT, Leinonen S et al (1996) Treatment of symptomatic congenital hepatic cysts with single-session percutaneous drainage and ethanol sclerosis: technique and outcome. $\mathrm{J}$ Vasc Interv Radiol 7:235-239

8. Drenth JP, Chrispijn M, Nagorney DM, Kamath PS, Torres VE (2010) Medical and surgical treatment options for polycystic liver disease. Hepatology 52:2223-2230

9. Goldstein HM, Carlyle DR, Nelson RS (1976) Treatment of symptomatic hepatic cyst by percutaneous instillation of Pantopaque. AJR Am J Roentgenol 127:850-853

10. vanSonnenberg E, Wroblicka JT, D'Agostino HB et al (1994) Symptomatic hepatic cysts: percutaneous drainage and sclerosis. Radiology 190:387-392

11. van Keimpema L, de Koning DB, Strijk SP, Drenth JP (2008) Aspiration-sclerotherapy results in effective control of liver volume in patients with liver cysts. Dig Dis Sci 53:2251-2257

12. Jusufovic R, Zerem E (2011) Percutaneous treatment of symptomatic non-parasitic benign liver cysts with $20 \% \mathrm{NaCl}$ solution. Med Arh 65:35-37

13. Benzimra J, Ronot M, Fuks D et al (2014) Hepatic cysts treated with percutaneous ethanol sclerotherapy: time to extend the indications to haemorrhagic cysts and polycystic liver disease. Eur Radiol 24:1030-1038

14. Zerem E, Imamovic G, Omerovic S (2008) Percutaneous treatment of symptomatic non-parasitic benign liver cysts: single-session alcohol sclerotherapy versus prolonged catheter drainage with negative pressure. Eur Radiol 18:400-406

15. Vachha B, Sun MR, Siewert B, Eisenberg RL (2011) Cystic lesions of the liver. AJR Am J Roentgenol 196:W355-W366

16. Vilgrain V, Silbermann O, Benhamou JP, Nahum H (1993) MR imaging in intracystic hemorrhage of simple hepatic cysts. Abdom Imaging 18:164-167

17. Bovenschen HJ, Janssen MJ, van Oijen MG, Laheij RJ, van Rossum LG, Jansen JB (2006) Evaluation of a gastrointestinal symptoms questionnaire. Dig Dis Sci 51:1509-1515

18. Yan-Hong F, Lin-Xue Q, Hai-Ma G, Qing Z, Yu G, Xiangdong H (2012) Sclerotherapy of simple hepatic cysts by repeated aspiration and alcohol instillation. Turk J Gastroenterol 23:359-365

19. Gebel M, Schulz M, Martin S (1990) Ergebnisse der transkutanen Sklerosierungstherapie von Leberzysten mit Polidocanol. In: Staubesand J, Schöpf E (eds) Neuere Aspekte der Sklerosierungstherapie. Springer, Berlin, pp 185-189

20. Yang CF, Liang HL, Pan HB et al (2006) Single-session prolonged alcohol-retention sclerotherapy for large hepatic cysts. AJR Am J Roentgenol 187:940-943

21. Yu JH, Du Y, Li Y et al (2014) Effectiveness of CT-guided sclerotherapy with estimated ethanol concentration for treatment of symptomatic simple hepatic cysts. Clin Res Hepatol Gastroenterol 38: 190-194

22. Montorsi M, Torzilli G, Fumagalli U et al (1994) Percutaneous alcohol sclerotherapy of simple hepatic cysts. Results from a multicentre survey in Italy. HPB Surg 8:89-94

23. Larssen TB, Viste A, Jensen DK, Sondenaa K, Rokke O, Horn A (1997) Single-session alcohol sclerotherapy in benign symptomatic hepatic cysts. Acta Radiol 38:993-997

24. Larssen TB, Rosendahl K, Horn A, Jensen DK, Rorvik J (2003) Single-session alcohol sclerotherapy in symptomatic benign hepatic cysts performed with a time of exposure to alcohol of $10 \mathrm{~min}$ : initial results. Eur Radiol 13:2627-2632

25. Lee S, Seo DW, Paik WH et al (2014) Ethanol lavage of huge hepatic cysts by using EUS guidance and a percutaneous approach. Gastrointest Endosc. doi:10.1016/j.gie.2014.03.037

26. Okano A, Hajiro K, Takakuwa H, Nishio A (2000) Alcohol sclerotherapy of hepatic cysts: its effect in relation to ethanol concentration. Hepatol Res 17:179-184

27. Van Keimpema L, De Koning DB, Van Hoek B et al (2011) Patients with isolated polycystic liver disease referred to liver centres: clinical characterization of 137 cases. Liver Int 31:92-98

28. Hahn ST, Han SY, Yun EH et al (2008) Recurrence after percutaneous ethanol ablation of simple hepatic, renal, and splenic cysts: is it true recurrence requiring an additional treatment? Acta Radiol 49: 982-986

29. Larssen TB, Rorvik J, Horn A et al (2004) Biochemical and cytologic analysis of cystic contents in benign non-parasitic symptomatic hepatic cysts before and after ethanol sclerotherapy. Acta Radiol 45:504-509

30. Bean WJ, Rodan BA (1985) Hepatic cysts: treatment with alcohol. AJR Am J Roentgenol 144:237-241 